\title{
Supporting Information for Understanding the Adsorption of Noble Gases in Metal-Organic Frameworks Using Diffuse Reflectance Infrared Fourier Transform Spectroscopy
}

\author{
Jian Liu ${ }^{\dagger, *}$, Praveen K. Thallapally ${ }^{\llbracket, *}$, and Thomas A. Blake \\ Energy and Environment Directorate, Pacific Northwest National Laboratory, Richland, \\ WA 99352, USA. \\ TPhysical and Computational Science Directorate, Pacific Northwest National \\ Laboratory, Richland, WA 99352, USA. \\ *To whom correspondence should be addressed. Fax: (509) 371-7249; Tel: 509-372-4477; \\ E-mail: Jian.Liu@pnnl.gov; Praveen.Thallapally@pnnl.gov.
}

Figure S1 A picture of the DRIFTS high-temperature reaction chamber.

Figure S2 Full DRIFTS spectra for Xe adsorption in the HKUST-1 at different pressures.

Figure S3 Full DRIFTS spectra for Xe adsorption in the Ni/DOBDC with a trace amount of pre-adsorbed water.

Figure S4 Powder XRD pattern for the Ni/DOBDC and HKUST-1. 


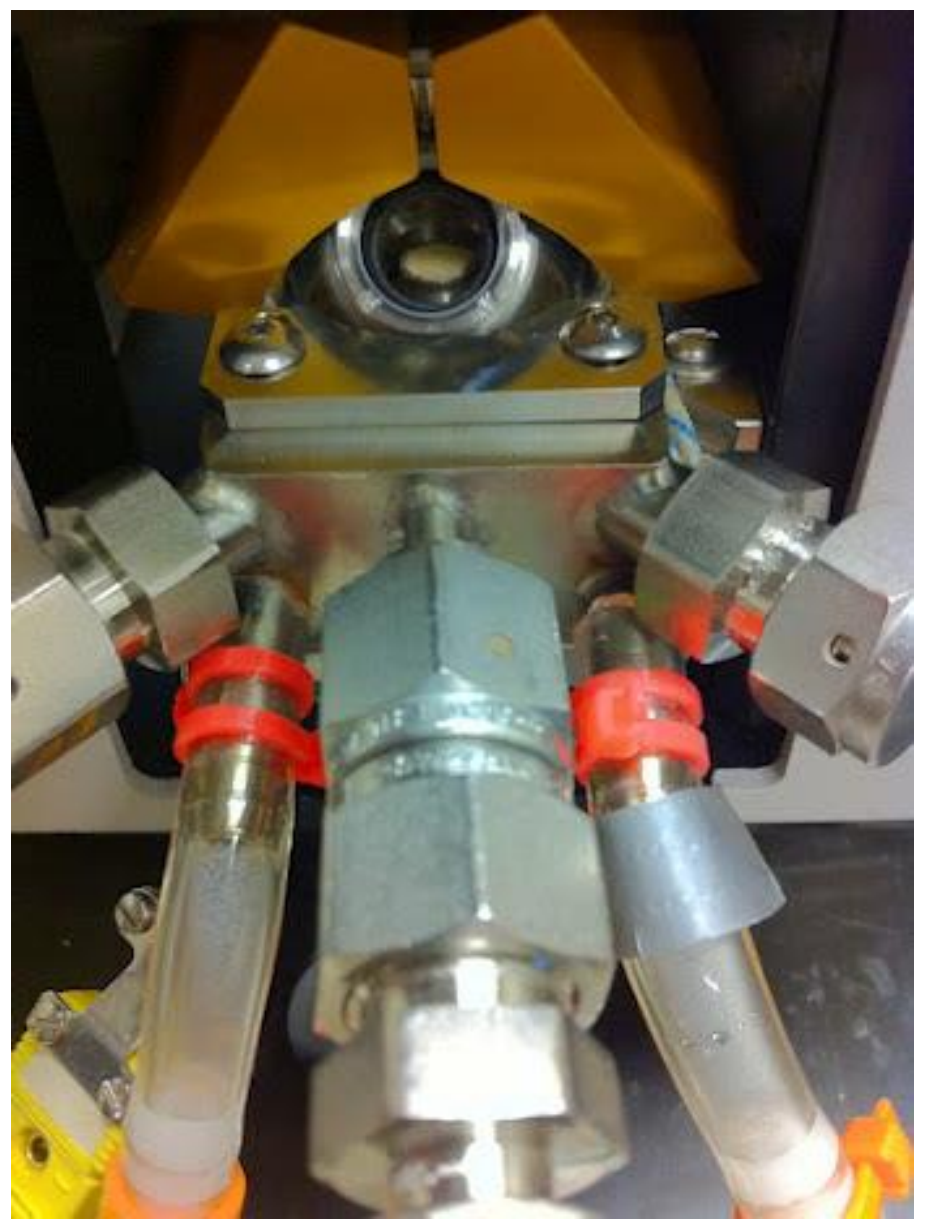

Figure S1. A picture of the DRIFTS high-temperature reaction chamber. The light-yellow powder sample can be seen through the circular UV window in the reaction chamber's dome. The off-axis ellipsoidal mirrors of the Praying Mantis can be seen on either side of the reaction chamber dome. The gas/vacuum and cooling water connections can be seen in the foreground. 


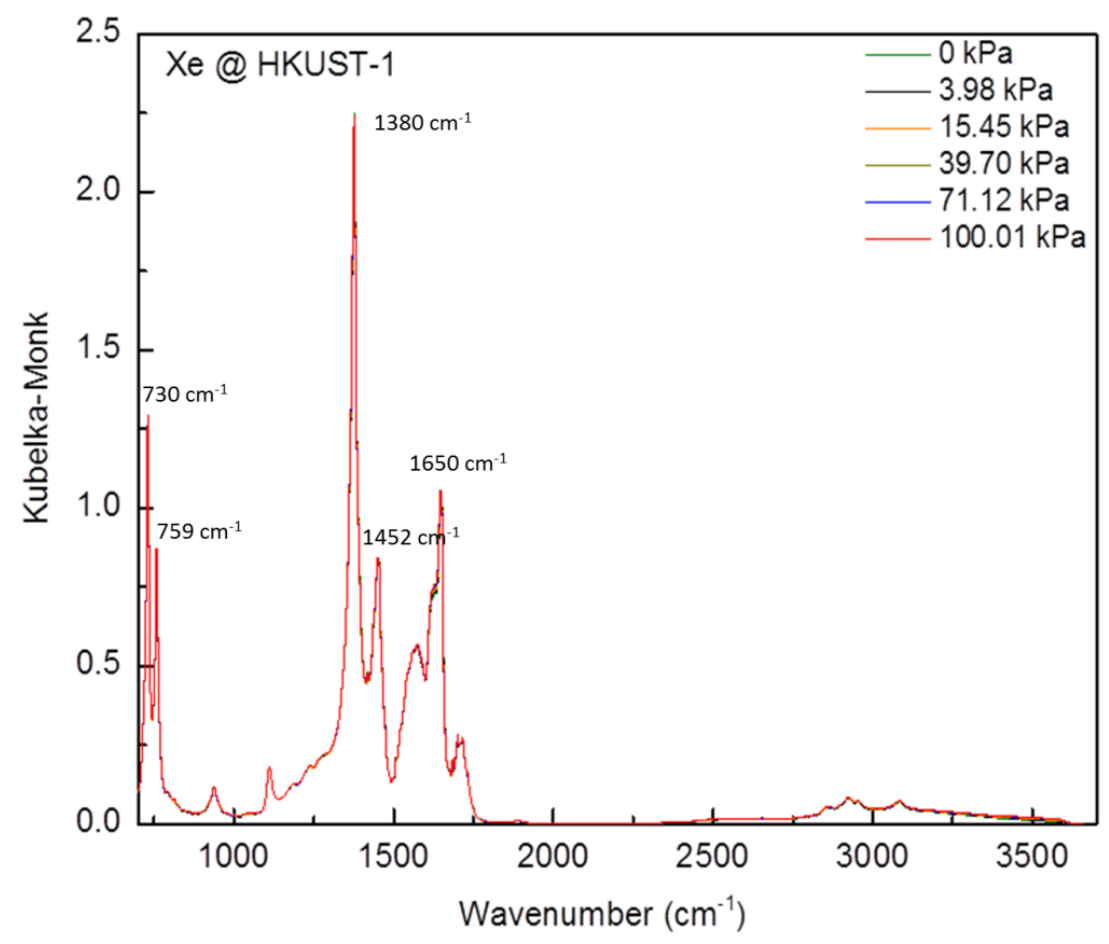

Figure S2. Full DRIFTS spectra for Xe adsorption in the HKUST-1 at different pressures. Peaks at 730 $\mathrm{cm}^{-1}$ and $759 \mathrm{~cm}^{-1}$ are from the out-of-plane C-H bending; The peak at $1380 \mathrm{~cm}^{-1}$ is from the symmetric COO stretch; Peaks at $1452 \mathrm{~cm}^{-1}$ and $1650 \mathrm{~cm}^{-1}$ are from the asymmetric $\mathrm{COO}$ stretch. 


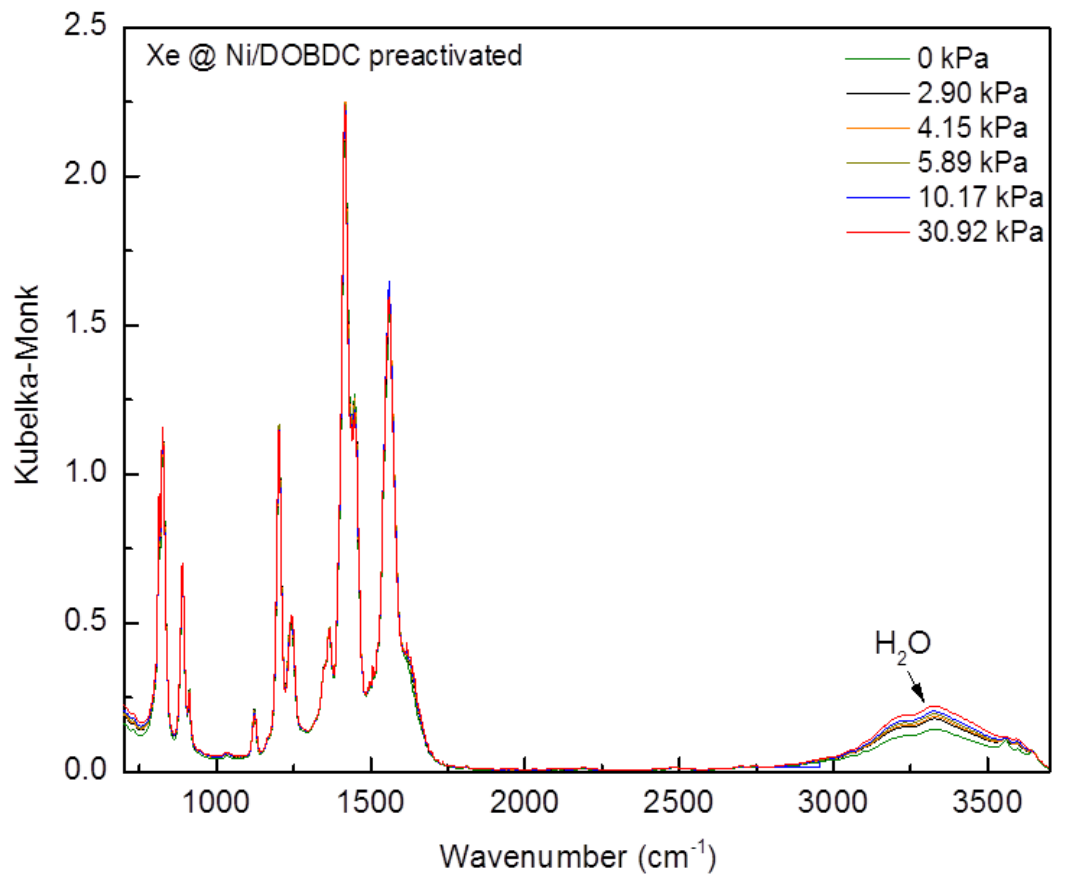

Figure S3. Full DRIFTS spectra for Xe adsorption in the Ni/DOBDC with trace amount of pre-adsorbed water. 

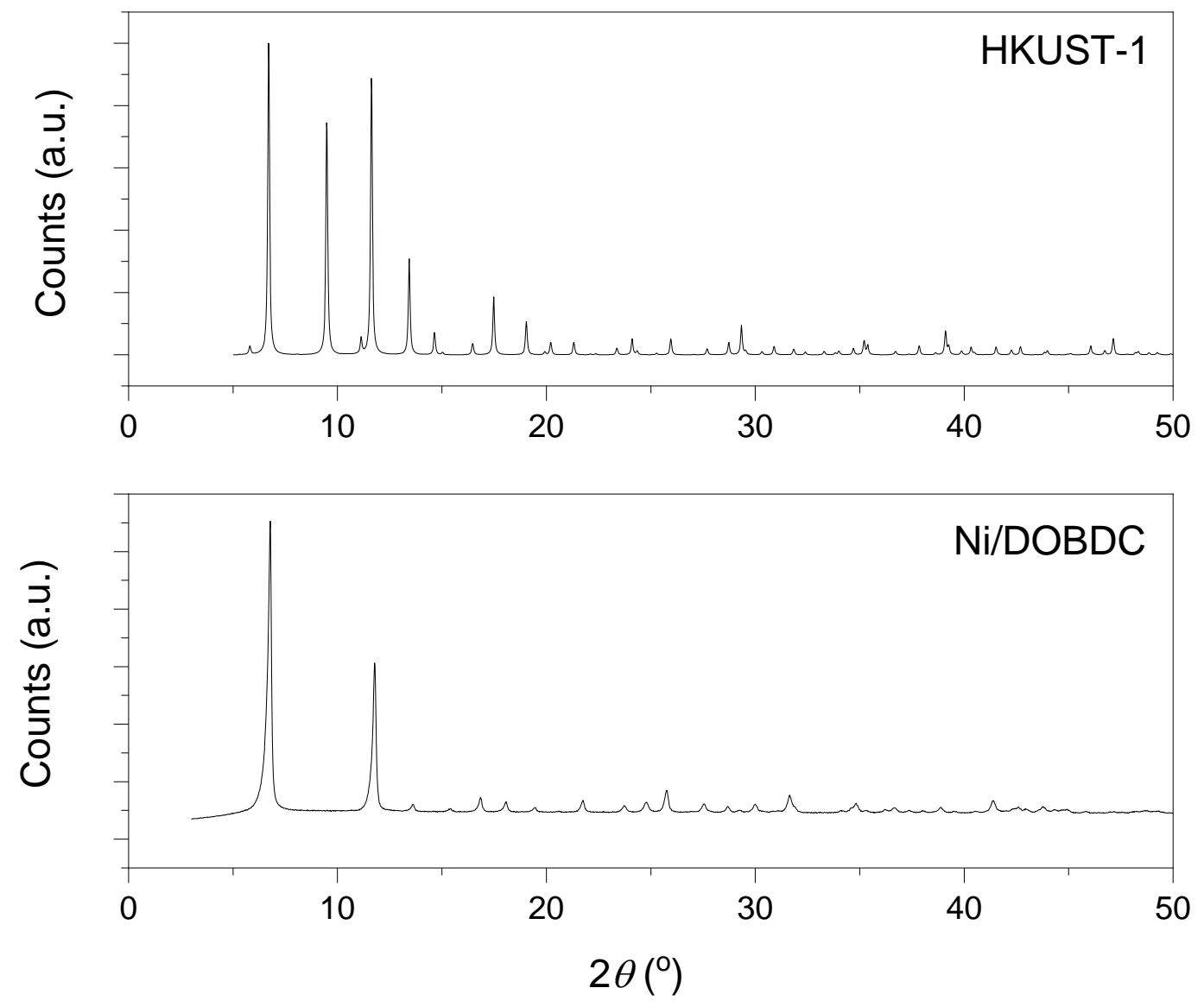

Figure S4. Powder XRD pattern for the Ni/DOBDC and HKUST-1. 